\title{
Belphégor
}

\section{Benoît Glaude, "Aire libre », Art libre ? Étude de la narration dans le champ de la bande dessinée franco- belge contemporaine}

\section{Vittorio Frigerio}

\section{(2) OpenEdition}

Journals

Édition électronique

URL : http://journals.openedition.org/belphegor/481

DOI : 10.4000/belphegor.481

ISSN : 1499-7185

Éditeur

LPCM

Référence électronique

Vittorio Frigerio, "Benoît Glaude, "Aire libre ", Art libre ? Étude de la narration dans le champ de la bande dessinée franco-belge contemporaine », Belphégor [En ligne], 12-1 | 2014, mis en ligne le 12 juin 2014, consulté le 22 septembre 2020. URL : http://journals.openedition.org/belphegor/481 ; DOI : https:// doi.org/10.4000/belphegor.481

Ce document a été généré automatiquement le 22 septembre 2020.

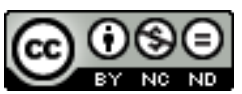

Belphégor est mis à disposition selon les termes de la Licence Creative Commons Attribution - Pas d'Utilisation Commerciale - Pas de Modification 4.0 International. 
Benoît Glaude, "Aire libre ", Art libre? Étude de la narration dans le champ de la bande dessinée francobelge contemporaine

Vittorio Frigerio

\section{RÉFÉRENCE}

Glaude, Benoît. "Aire libre », Art libre ? Étude de la narration dans le champ de la bande dessinée franco-belge contemporaine. Louvain-la-Neuve : L'Harmattan/Academia S.A., 2011. ISBN : 978-2-8061-0017-7 
1 Le soi-disant « neuvième art » (on oublie bien souvent qu'il vient après la télévision, en huitième position) est en train de passer par ce processus de consécration critique qui marque d'habitude un tournant dangereux pour les productions culturelles de masse, tant il semble se produire toujours en même temps qu'une désaffection grandissante de ce public enthousiaste et relativement peu exigeant qui avait fait sa fortune. Les grandes revues de bande dessinée qui ont fait le succès du médium dans l'aprèsguerre et jusque dans les années 80 n'existent plus - à l'exception d'un Journal de Spirou qui ne peut plus guère prétendre au poids qu'il avait jadis - et alors que la production d'albums montre encore ce qui semble être les signes d'une santé florissante, peu de personnages paraissent susceptibles d'atteindre à l'avenir au statut iconique des grands ancêtres signés Hergé, Jacobs ou Martin, et le séjour des nouvelles séries sur les rayons des librairies est souvent d'une brièveté quelque peu inquiétante.

2 Le succès d'estime, et plus rarement économique (voir Persépolis) des productions contemporaines qui se situent en opposition à un modèle commercial perçu comme dépassé et banalement répétitif, a suscité chez les éditeurs traditionnels le désir de tenter de récupérer le terrain perdu et de créer des collections réservées à des œuvres plus originales, l'espoir non déguisé étant aussi de faire (re)venir au bercail les signatures les plus en vue de cette nouvelle vague de BD «d'auteur », ou d'éviter des dérapages éventuels de la part d'auteurs plus « classiques » en quête d'une plus grande liberté créatrice.

3 « Aire libre » est une telle tentative de la part d'un des éditeurs historiques de la bande dessinée belge, Dupuis. Le titre de la collection - qui laisse malencontreusement supposer que le restant des espaces mis par Dupuis à la disposition de ses créateurs n'est guère à l'enseigne du même principe - l'indique bien comme un domaine privilégié pour une analyse qui voudrait identifier le montant précis d'originalité, d'autonomie créatrice et de nouveauté présents dans ces albums. C'est là la tâche que se donne, de façon fort systématique, Benoît Glaude, en passant en revue divers albums parus sous ce label : Vitesse moderne (de Blutch et Ruby), Agadamgorodok (de Bailly et Lapière), Les Enfants (de Stassen), et en plus Total Jazz de Blutch, paru au Seuil, et La Coupe Castar (de Bailly, Francesca, Lapière et Mathy), paru encore chez Dupuis, mais dans la collection «Tous publics ». Cela avec force tableaux et à l'aide d'une méthodologie critique relevant de la narratologie et de la sémiotique, s'appuyant en particulier sur les travaux de Pierre Fresnault-Deruelle, non sans passer parfois du côté de Jacques Dubois et de celui de Jean-Louis Tilleuil, avec une allusion occasionnelle à Roland Barthes. 
4 La discussion de chaque album se fait à la fois au niveau de l'image et à celui des textes, la lecture de l'image privilégiant la planche comme unité narrative de base, examinée comme espace narratif tout aussi bien que d'un point de vue «tabulaire " (la planche en tant que tableau), deux modes de perception coexistants. L'organisation très systématique de la réflexion, renforcée par l'utilisation régulière de tableaux, reflète une approche qui fait montre d'une méfiance certaine pour l'idée même de spontanéité, que le critique ne cesse de baliser. À la suite notamment de déclarations de Blutch, qui se pose en auteur instinctif, pas du tout intellectuel, Glaude n'hésite pas à rebondir en affirmant: «Sans douter de la bonne foi de ces déclarations, je décrirai les procédés narratifs qui produisent cette spontanéité évidente s'accordant avec les intentions de l'auteur » (77-8). L'analyse qu'il fournit est, il faut le dire, convaincante, quoique de certaines manières prévisible. Par des voies différentes, il arrive à reformuler une tripartition du champ de production de la bande dessinée en «art moyen ", « art bourgeois » et "avant-garde » (113) qui reproduit en gros la célèbre tripartition de la culture de Dwight MacDonald dans Against the American Grain en « lowbrow", « middlebrow", « highbrow".

5 Le discours se poursuit par une identification progressive des tendances de la bande dessinée d'auteur contemporaine à reproduire le schéma qui avait porté les tenants de l'art pour l'art à prendre leurs distances d'un romantisme perçu comme avili par sa trop grande proximité à la masse d'un lectorat peu exigeant - l'illustration concrète étant la politique de production limitée de l'éditeur de Total Jazz de Blutch, Le Seuil, qui contribue « au positionnement avant-gardiste de Seuil B.D. au sein du champ de la bande dessinée» (121). Quant à Aire Libre, positionnée au "pôle neutre de la production bédéique » (137), elle arrive à concilier consécration bourgeoise et profits économiques, en plus d'un degré de reconnaissance culturelle normalement hors d'atteinte pour un grand éditeur commercial. Le capital symbolique s'unit ainsi au capital tout court dans la recherche d'une reconnaissance culturelle qu'une activité régulière sur vingt-cinq ans et plus parvient à consacrer. Tout cela, évidemment, sans qu'il soit nullement question d'«art libre ", mais bien d'un mouvement fortement conditionné à l'intérieur de schémas où l'intention autonome de l'auteur jouit d'espaces bien moins vastes qu'il n'a parfois tendance à le croire.

6 La répartition des cinq albums étudiés en groupes, selon qu'ils appartiennent à l'avantgarde, l'art bourgeois, ou l'art moyen, permet au critique de noter comment leur position dans le champ actuel est redevable de phénomènes identiques à ceux qui opèrent dans le champ de la littérature. La conclusion est que « dans le champ de la bande dessinée, chaque instance de production (auteur-éditeur) a à se situer par rapport aux trois positions principales et elle répond aux attentes du public particulier que cette place lui destine » (137).

7 Comme c'est, ma foi, souvent le cas pour des textes marqués au coin de la théorie narratologique, ce volume ne se lit pas toujours avec la plus grande aisance, ni même parfois avec beaucoup de plaisir. Le sérieux de la démarche et la finesse de l'analyse compensent toutefois ces faiblesses probablement inévitables et font de ce livre une contribution très intéressante aux études contemporaines sur la bande dessinée francobelge. 


\section{AUTEURS}

VITTORIO FRIGERIO

Dalhousie University 